Chapter 12

\title{
Shape Memory Polymers for Smart Textile Applications
}

\author{
Suman Thakur
}

Additional information is available at the end of the chapter

http://dx.doi.org/10.5772/intechopen.69742

\begin{abstract}
Shape memory polymer (SMP) is a genre of smart materials which demonstrate a capability to fix in the temporarily programed shape and to recover the original shape upon exposure to external stimuli. Such unique and amazing properties of SMPs are applied to develop smart textiles by incorporating SMP into the fabric. Various types of stimuliresponsive SMPs such as thermal, light, $\mathrm{pH}$, and moisture have been used in textiles for enhancing or attaining some smart properties. The integration of SMPs in fabric provides many fascinating and improved properties such as good aesthetic appeal, comfort, textile soft display, smart controlled drug release, fantasy design, wound monitoring, smart wetting properties and protection against extreme variations in environmental conditions. In this chapter, we discussed different types of SMP used for this purpose, their functions, and applications in textiles with their potential applications in the near future.
\end{abstract}

Keywords: shape memory polymers, stimuli-responsive materials, smart textile

\section{Introduction}

Shape memory polymer (SMP) is a genre of stimuli-responsive materials with ability to fix a programed shape and to return from the deformed shape to its original permanent shape while induced by an external stimulus such as heat, light, humidity, electric field, magnetic energy, pH and so on (Figure 1) [1-4]. This amazing property of SMPs has inspired many researchers and industrialists to develop smart textiles by incorporating them into the fabric. Nowadays, SMPs carved an attention for use in textile where they strongly respond to changes in heat and moisture levels, ensuring greater comfort for the wearer. Incorporation of SMPs also provides some smart features along with comforts such as aesthetic appeal, textile soft display, smart-controlled drug release, wound monitoring, smart wetting properties, and protection against extreme variations in environmental conditions. Therefore, smart functional textiles have developed swiftly in the past few decades [5-7]. Digital components, 


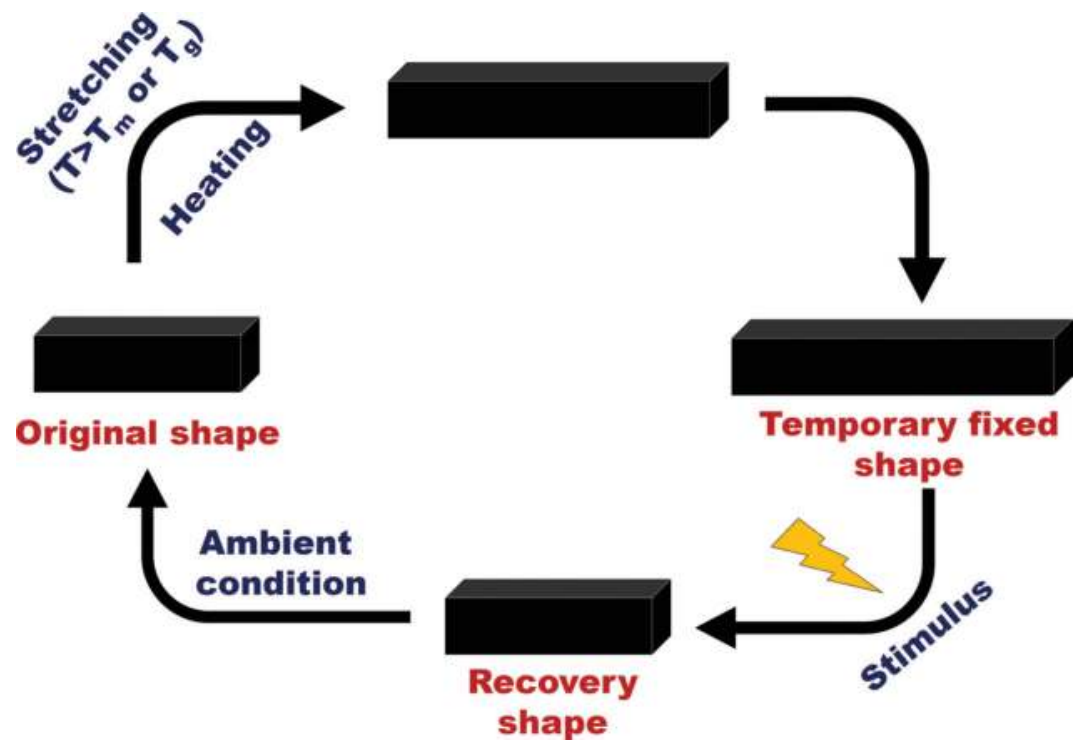

Figure 1. The schematic representation of shape memory behavior of SMPs.

computing, and electronics embedded e-textiles offered many novel functions to garments [8-10]. Various new and unique functions containing textiles are developed such as luminescent textiles, textile displays, emotion sensing dresses, self-cleaning textiles, temperatureregulated textiles, and self-moving textiles [11-14].

Different SMPs such as shape memory polyurethane (SMPU), polyester, poly-hydroxyproline, polysilamine, etc., and some responsive hydrogels including poly( $\mathrm{N}$-isopropylacrylamide) (PNIPAAm) hydrogels, polythiophene gel, etc., are used to fabricate such smart textile. In addition to that, various SMP composites also prepared for different end applications. SMPs and their composites have several advantages for textile coatings:

(a) Different stimuli can be used which textiles commonly undergo, such as water, light, and heat

(b) They have highly flexible programming, which can be triggered by various stimuli by single- or multiple-step processes

(c) They exhibit tunable properties that can be engineered easily to be applied onto the fabric surface

(d) They have a light and adjustable modulus that is easily identical to a textile's softness

In this chapter, we discussed the different types of SMPs used for smart textile, their functions, and especially applications of such smart textile with their potential applications in the near future. 


\section{Function of shape memory polymers in textiles}

SMPs possess different versatile functions. The following contents introduce such functions of SMPs which use for the fabrication of smart textile.

\subsection{Thermal and moisture controlling}

Smart breathable garment is one of the most desired products in textile sector, which can be fabricated using SMPs. This garment can regulate the transfer of heat and moisture to wearer's body. The water vapor permeability (WVP) of the SMPs regulates with the human body temperature. The molecular free volume of the SMPs significantly increases when the body temperature is above the glass transition temperature $\left(\mathrm{T}_{\mathrm{g}}\right)$ of SMPs. This aids the transfer of vapor and heat through perspiration for comfort in wearer's body. Again, the molecular free volume decreases when the body temperature is below $\mathrm{T}_{\mathrm{g}}$ of the SMPs. This restricts the both heat loss and the moisture to pass through. Thus, the SMP is a good choice for maintaining stable body temperature. Textiles and garments containing SMPs are still being developed and investigated. Lamination, coating, knitting, and weaving are used to integrate SMPs into textiles [15].

In this regards, Wang et al. fabricated a garment which afforded thermal protection in too cold conditions [16]. In addition to that, Crespy and Rossi also incorporated the thermalresponsive hydrogels in textiles for smart heat and moisture management [17]. Kim et al. found that PNIPAAm-grafted polypropylene nonwovens possessed good thermo-responsive WVP [18]. SMPU-coated fabrics demonstrated a rapid increase in WVP at transition temperature. Additionally, Mondal and $\mathrm{Hu}$ fabricated a cotton fabric by using SMPUs containing a small percentage of multiwalled carbon nanotubes (MWNTs). This fabricated cotton demonstrated excellent WVP with good UV protectability [19].

Along with this, few commercial products also came into market. In this context, Mitsubishi Heavy Industries fabricated a SMPU membrane namely 'DiAPLEX-The intelligent material' [20]. It provides some special properties such as waterproofed, windproofed and breathability. Its working mechanism is based on the principle of thermal vibrations. These vibrations are generated by micro-Brownian movements of polymeric chains. SMPs can be attached in form of a laminate to a variety of fabrics, being capable of adapting to the surrounding environmental conditions (Figure 2). In addition, Toray Industries and Marmot Mountain Works ${ }^{\circledR}$ also developed a SMPU film MemBrain ${ }^{\circledR}$ laminated between adjacent layers of clothing. This fabricated cloth also demonstrates waterproof and breathable functions [20]. SMPU film responds when the temperature of the outer layer of clothing is sufficiently down; therefore, the air gap between the layers of clothing becomes broader. At low temperature, this airgap broadening is attained if the film changes an out-of-plane deformation. This deformation need be adequately strong to resist the weight of the clothing, and the forces induced by the movements of the wearer. Again, the deformation should be reversal when the temperature of outer layer is subsequently hot. In addition to that, Apollo dress shirt from Ministry of Supply uses NASA space suit technology, which can also regulate body temperature and 


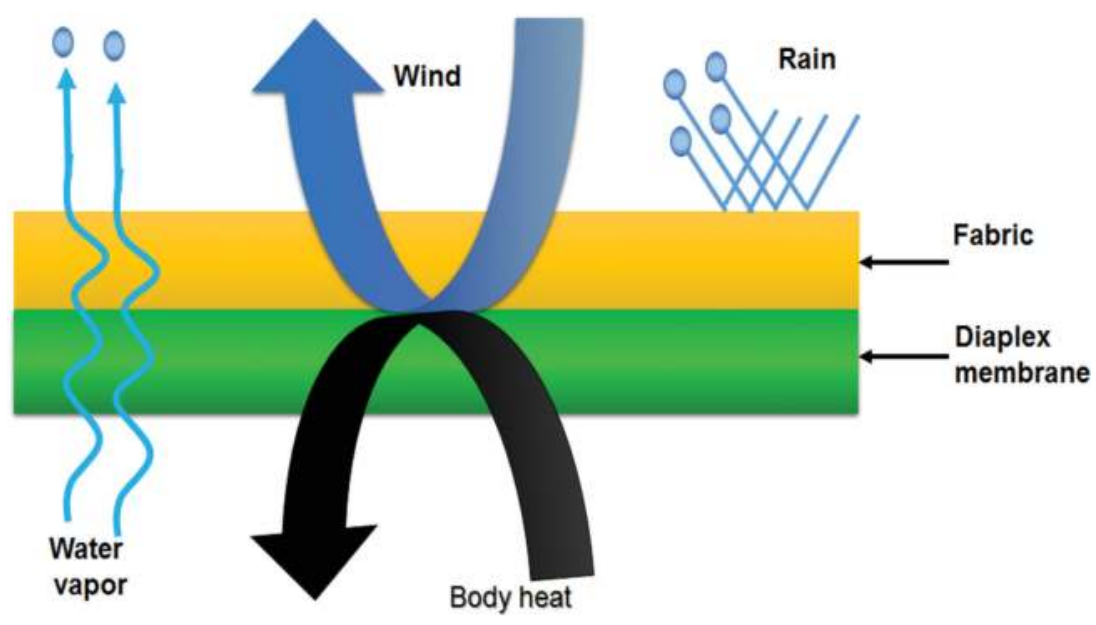

Figure 2. Structural illustration of twin layer of DiaPlex fabric.

also keeps wearers dry and odor free. Additionally, various types of thermo-regulated textile products, such as blankets, sleeping bags, underwear, jackets, sports garments, socks, ski boots, helmets, etc., have been on the market.

\subsection{Self-adaptability of shape}

SMP fibers are utilized to produce self-adaptable textiles which can easily regulate its structures with changes in environmental temperature. Though the shape memory effect (SME) of a fiber is basically considered as the change of its length, but this SME can take a range of forms after incorporated into fabrics, such as bending, shrinkage, and thickness increase, which is determined by the fabric structures. The SMP fibers fabricated garments can be properly expanded to fit in the wearer's body. Vertical pressure tests suggest that the garments made of SMP fibers possess a relatively low vertical tension stress in comparison with elastic fibers. This can be attributed to the deformability and fixability of SMP fibers into temporary shapes, which diminishes the adverse pressure sensation to wearers. The garment made of SMP fibers can enlarge and adapt to the wearer size, while no significant pressure is being exerted on the wearer due to the shape fixity of the SMP fibers. The garment made of Spandex fibers generates pressure on the wearer due to the high elasticity of the Spandex fibers in the fabric. The fabric made with SMP fibers with improved comfort sensation can be used especially for intimate apparel and low-pressure socks [21, 22].

\subsection{Shape retention}

The cotton fabrics treated with SMP possess a wrinkle-free effect [23]. In fabrics, especially cotton materials, wrinkles are easily generated during wearing or storage due to the debonding and slippage of hydrogen bonds. In order to avoid that wrinkles in cotton, dimethyloldihydroxyethylene urea (DMDHEU) is mostly used during finishing of cotton. The SMP-treated fabric does not release formaldehyde compared to DMDHEU, which is a benefit of usage 
of SMP. In addition to that SMPU finishing on cotton improves the mechanical strength of fabrics to some extent in contrast with that of conventional durable-press finishing process. The SMPU emulsion-treated fabric demonstrated adequate wrinkle-free effect after repeated washing, and it can easily last up to hundreds of laundering cycles [20]. SMPU-treated cotton fabrics also possess a greater crease and pattern retention ability due to presence of SME. The crease or pattern design on textiles can provide the esthetic appeal in garment. In addition to cotton, wool fabrics were also treated with the SMPU emulsion. SMP-treated wool garment has better dimensional stability than that of an untreated garment. This is due to the fact that SMPU covers wool fiber, and therefore, it reduces the wool directional frictional effect after the finishing process. The untreated wool garment shrinks to a small size, while the treated garment maintains its size after laundering. Wool fabrics and sweaters possess a daunting tendency to felting with the entanglement of scales by directional friction. The treated wool fabric showed significant reduction in felting.

\subsection{Smart wettability}

Smart wettability is one of the most desire requirements in garment sector. In most of the cases, it can be achieved by providing the hydrophobic surface on the fabric. Such surface with a water contact angle greater than $150^{\circ}$ and a sliding angle (SA) lower than $10^{\circ}$ can generally be obtained by periodic microsized or nanosized patterns. In addition to microparticle or nanoparticle coating or finishing on the textile surface, SMP coating provides the same effects. The micro- or nanopattern effect can be achieved by the phase transformation of SMPs. If a SMP coating is used on a fiber surface, it will demonstrate different shrinkage effects after heating and cooling. This reflects that SMP-coated fabric has a potential application to achieve a water-repellent effect or control water-spreading behavior. Besides hydrophobicity, controlling the movement of water on textile surfaces also attracts interest of many researchers. The behavior of water reverting from repellency (hydrophobic) to adhesion (hydrophilic) is basically governed by a combination of the contact angle and sliding angle. In this regard, Zhong et al. fabricated smart cleaning cotton fabrics using cross-linked thermo-responsive polymer, poly(2-(2-methoxyethoxy) ethoxyethyl methacrylate-co-ethylene glycol methacrylate) [24]. They found that both the wetting time and contact angle of the cotton fabrics significantly increased, when the temperature is above the lower critical solution temperature (LCST), indicating the cotton surface switches from hydrophilicity to hydrophobicity. As the performance of cleaning is generally enhanced when the surface is more hydrophilic, the cotton fabrics with cross-linked polymer can be used to prepare the fabrics with smart cleaning ability. Such fabrics can be cleaned at much lower temperatures as compared with common cotton fabrics. This can efficiently save a significant amount of energy which is in textile cleaning.

\section{Different type SMPs used in textile application}

\subsection{Thermal-responsive SMPs}

Thermal-responsive SMPs are one of the most extensively studied and used SMPs. Various forms of SMPs such as solution, emulsion, film, fiber, foam, and bulk forms are developed 
for suitable application under different circumstances. Generally, $\mathrm{T}_{\mathrm{g}}$ or melting temperature $\left(\mathrm{T}_{\mathrm{m}}\right)$ is selected as triggering temperature of this kind of SMPs. The triggering temperature of SMPs is adjustable and can be adjust around body temperature too. Several advantages of these SMPs make them suitable for textile applications such as superior processability, mechanical properties, high deformability, and high recoverability. Yang et al. developed an $N$-isopropylacrylamide (NIPAAm) hydrogel-treated cotton fabric that was able to absorb a large amount of water from humid air, which can be subsequently released at the higher temperature than the LCST of the polymer. The combined effect of the intrinsic phase transition of PNIPAAm around the LCST and the change between extreme superhydrophilic/superhydrophobic morphologies of the hydrogel is mainly responsible for this phenomenon [25].

\subsection{Water/solvent-responsive SMPs}

Water or other solvent is used for triggering this kind of SMPs. The original shape is recovered due to the plasticizing effect of solvent molecules, which enhanced the flexibility of polymeric chains. If an SMP has a hydrophilic or water-soluble ingredient, the shape recovery can be accelerated. A pyridine unit, which is responsive to moisture, is good choice to use for improvement of the moisture absorption in PU. Hu et al introduced a pyridine unit ( $\mathrm{N}$-bis(2hydroxyethyl) isonicotinamine) into SMPU to achieve moisture-responsive SMPU with high strain recovery and recovery speed [26]. In addition to that, $\mathrm{Hu}$ and his team also fabricated cellulose nanowhiskers (CNWs)/elastomer and CNW/SMPU nanocomposites which exhibited good water sensitivity due to the reversible hydrogen bonding. They found that the fabricated CNW/SMPU showed triple SMEs upon exposure to sequential thermal and water stimuli [27].

\subsection{Thermal/pH-responsive polymeric hydrogels}

Thermal/pH-responsive polymeric hydrogel is another kind of SMPs which is used in textiles to produce smart textile. This polymeric hydrogel is a three-dimensional macromolecular gel network containing a large fraction of water within their structures. The degree of swelling of such gels increases or decreases at below or above a critical temperature. At a higher temperature, the hydrophobic interaction among hydrophobic segments is high, while the hydrogen bonding is less. The final result yields shrinkage of gel due to the hydrophobic interactions. PNIPAAm hydrogel is most frequently studied polymeric gel in textile. PNIPAAm hydrogel possesses a LCST in aqueous medium around $32-34^{\circ} \mathrm{C}$ which is close to the human body temperature. By adjusting the PNIPAAm copolymer composition and topology, the phase transition can be easily tuned. PNIPAAm hydrogel-coated fabrics showed reversible swelling/shrinkage (hydration/dehydration) and good water vapor permeability (WVP) of the fabrics. These properties enable the achievement of temperature-sensitive hygroscopic fabrics, temperature-sensitive deodorant fibers, and temperaturesensitive nutrient delivery fabrics. Yang et al. grafted PNIPAAm on cotton fabrics to cover the hydrophilic surface of the cotton with a polymer layer which would be able to absorb 
water from a humid environment, below the LCST, and release it upon a temperature change [25]. The coated cotton fabric shows temperature-triggered reversible and repeatable change in the wettability (Figure 3). Instead of such advantages of PNIPAAm, it possesses moderate cytotoxicity which hampers its application in few special field. Researchers have developed the copolymer composed of 2-(2-methoxyethoxy)ethyl methacrylate and oligo(ethylene glycol) methacrylate as an ideal substitute for PNIPAAm [28]. Lee and his team investigated the pH-responsive LCST behavior of polyethylene oxide-based functional polymers with different pendant amine groups and varying side chain lengths. Depending on the nature of the pendant amine groups (primary amine, dimethylamine, and diethylamine) and the hydrophobicity of the side chains (ethyl, propyl, and hexyl), LCST is easily tuned from 44 to $100^{\circ} \mathrm{C}$ under different $\mathrm{pH}$ conditions [28].

\subsection{Light-active polymers}

Light-active polymers are particularly attractive for various textile applications. Many light-active polymers have been synthesized and used in this field, such as azobenzenebased crystalline elastomer, anthracene-based polymers, and coumarin-based polymers. Even though some light-active threads and nanofibers have been reported, the application of light-active polymers in textiles is yet to explore comprehensively. Requirement of special wavelength light to activate such light-active polymers is the main restriction for their practical applications in garments. Esteves and coworkers fabricated a light-active polymer-coated cotton fabric by functionalizing a spiropyran-NIPAAm hydrogel [29]. This treated fabric is capable of dimensional changes upon irradiation with visible light or upon a temperature stimulus (Figure 4). This light active fiber may also pave an opportunities in other technological applications, such as breathable textiles and agricultural purposes.

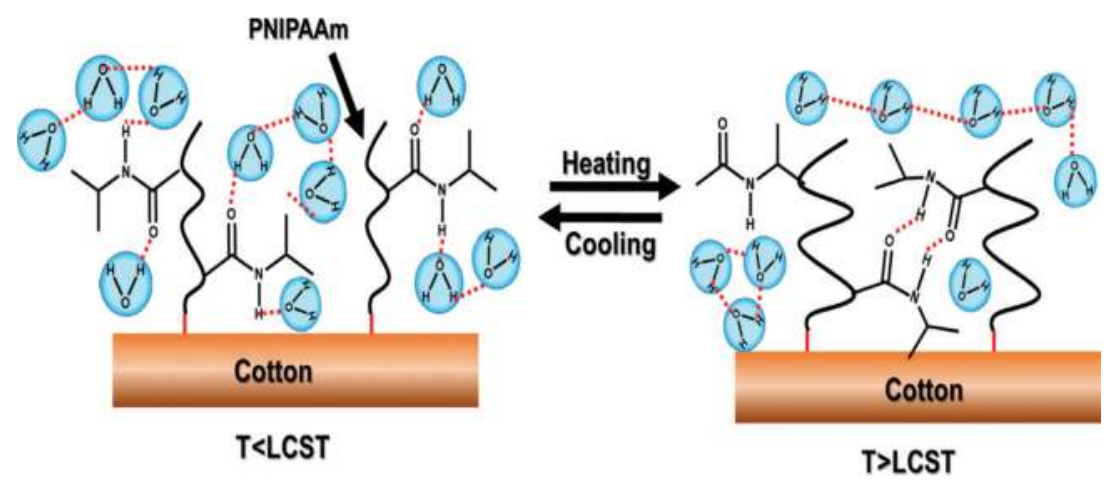

Figure 3. Temperature-triggered reversible wettability of PNIPAAm. 


\section{Application of SMPs in textile}

\subsection{Finishing fabrics}

Thermo-responsive SMPs can be applied in the textile via garment finishing. Wrinkle-free, crease retention, anti-shrinkage and dynamic aesthetic textiles are fabricated by treating SMPs on fabrics. The cotton fabrics treated with SMP show good wrinkle-free effect. Cotton fabric treated with SMPU can recover to its original flat shape (wrinkle-free) within a minute upon blowing steam over it (Figure 4a). SMP-treated fabrics possess good crease and pattern retention ability (Figure $\mathbf{4 b}$ ). As a result, good aesthetic appeal is achieved in such treated fabric.

\subsection{Breathable fabrics}

SMPUs are used for fabrication of breathable fabrics. WVP of the SMPU can be easily altered according to the wearer's body temperature. The overall WVP of SMPU films can be improved by incorporating hydrophilic segments such as dimethylpropionic acid and diol-terminated poly(ethylene oxide) in SMPUs. The overall WVP of SMPs can also be significantly increased by forming microfoams in the SMPUs.

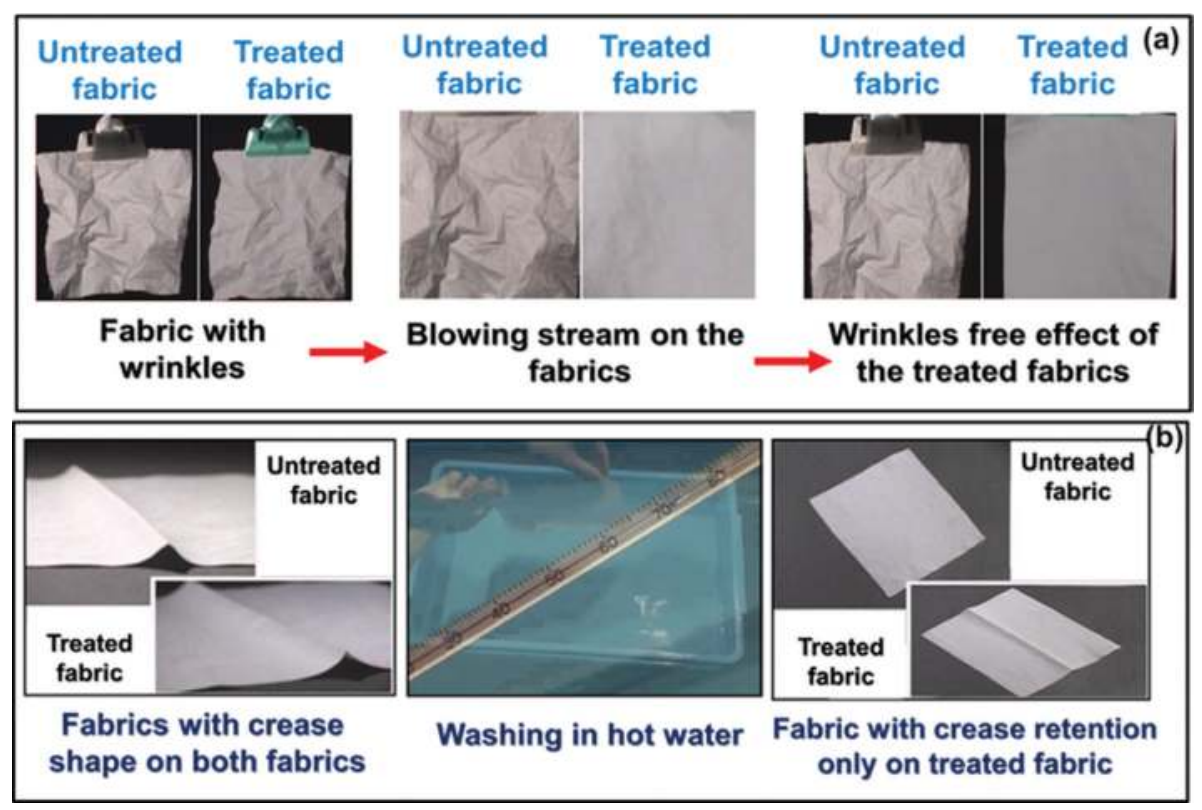

Figure 4. (a) Wrinkle-free effect and (b) crease and pattern retention ability of SMPU-treated cotton fabrics (reproduced with permission from Ref. [15]). 


\subsection{Damping fabrics}

SMPs can absorb impact energy due to their good damping properties at around $\mathrm{T}_{\mathrm{g}}$. Allied Signal Inc. manufactured an automotive seatbelt fabric using SMP fibers (Securus fibers) which can absorb the kinetic energy and so effectively increase a passenger's safety by utilizing the damping effect of the SMPs [30]. The Securus fibers are made of shape memory poly(ethylene terephthalate)-poly(caprolactone) block copolymers. It is reported that the fiber can absorb energy from the body's forward motion and thus improves the safety of passengers during an accident. At first, the seatbelt holds the passenger securely in place; then, it elongates slightly and cushions the body as the belt absorbs the force from the body.

\subsection{Skin-care products}

The skin-care products prepared using stimuli-responsive hydrogel-treated textiles possess moisturizing, whitening, brightening and even anti-ageing effects on human skins. Figure 5 demonstrates a facial mask which is made of nonwoven fabric treated with thermo-responsive polymeric gel [31]. This mask is responsive to human body temperature. Such smart mask can also utilize as a temperature-responsive carrier medium for controlled release of nutritious ingredients, perfumes, or other drugs to human skin. The study has revealed that the release of vitamin $C$ easily could be controlled just by judiciously changing the temperature [32]. Even though such advantages, there are still some challenges such as the high stiffness and brittleness that are important to be resolved.

\subsection{Wound-dressing products}

Chitin/chitosan and chitosan derivatives are extensively used to fabricate wound dressing product due to their good antibacterial properties and wound-healing effects. Chitosan hydrogel as

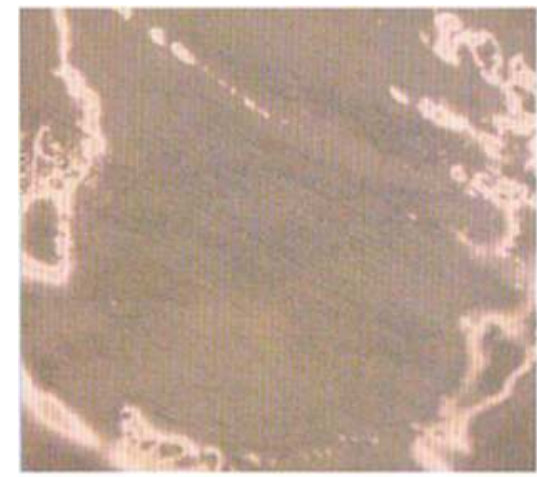

Room temperature

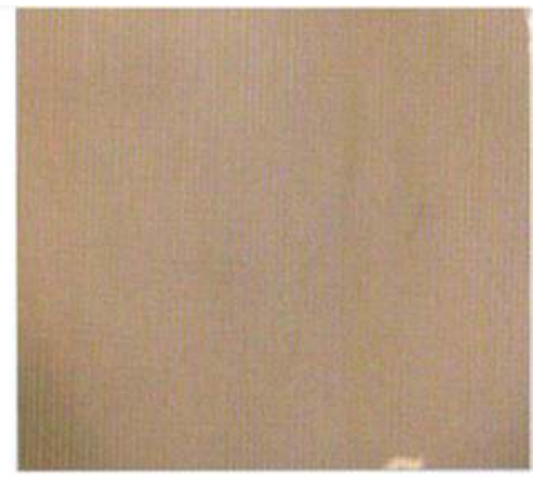

Body temperature

Figure 5. A facial mask made of the nonwoven fabric treated with TRPG at room temperature and body temperature (reproduced with permission from Ref. [15]). 
a wound dressing material can help in the reestablishing of skin architecture. Chitosan-treated alginate filaments and cotton fabric have been fabricated for advanced wound dressings [33]. In addition to chitin/chitosan, various biopolymer-based hydrogel products have also been developed for wound dressing $[34,35]$. Such smart wound dressing materials can deliver a novel drug release system in response to variations in $\mathrm{pH} /$ temperature; thus, the wounds can quickly heal.

\subsection{Deodorant fabrics}

Such special fabrics have an ability of releasing deodorant agents at certain temperatures. The smart fabric is fabricated by coating a stimuli-responsive polymeric hydrogel on the textile surface. The hydrogel is attached to the textiles surface through chemically cross-linking by using a functional monomer, such as acrylamide, and a cross-linking agent such as 2-(diethylamino)ethyl acrylate. Deodorant is generally incorporated into the hydrogel during or after the cross-linking reaction. Usually, $\beta$-cyclodextrin is loaded with polymeric gel for further enhancing the controlled release properties of hydrogel-modified textiles [36]. $\beta$-cyclodextrin has a hydrophobic interior cavity and a hydrophilic external surface in its structure [37]. Therefore, different types of hydrophobic guest deodorant moieties can be included in its cavity. Recently, a few body-responsive deodorant hydrogel products are also available on the market. Even though such advantages of deodorant fabrics, it is not fully explored. Few issues such as soft handle and high stability of the products need to be taken into consideration for development of such fabric. Another important factor is controlling of the hydration degree of the product in the open environment.

\subsection{Smart energy storage textiles}

Wearable electronics carve a distinct niche to our daily life by providing changes in outdated living habits to less consuming and healthier ones. Electronic circuits are generally integrated into flexible textile matrices to achieve such smart wearable electronics. Batteries and supercapacitors (SCs) are two archetypal energy storage devices, which is extensively used in our daily life. The capability of storing energy strongly relies on the properties of electrodes, including microarchitecture, conductivity, and specific surface area. SMPs have been applied in SCs to provide SME into SCs. SMPs in a SC usually serve as the substrate or core, with a conducting layer and an active layer attached successively to the surface of the shape-memory substrate or core to make a shape memory electrode. Huang et al. used shape memory material as the fiber core, which was consecutively coated with $\mathrm{MnO}_{2}$ and $\mathrm{PPy}$ to fabricate temperature-sensitive electrodes [38]. Two of the such fabricated electrodes are twisted to make a capacitor with a specific capacitance of $198.2 \mathrm{~F} \mathrm{~g}^{-1}$ at a current density of $1 \mathrm{~A} \mathrm{~g}^{-1}$ for 20,000 cycles. They use shape memory material as the main skeleton to make wireshaped supercapacitor possess a unique shape memory effect which has, indeed, a practical significance when it comes to risk management (Figure 6).

Deng et al. also developed a fiber-shaped supercapacitor (FSSC) by winding aligned carbon nanotube sheets on a SMPU substrate [39]. The fabricated FSSC can be transformed and "frozen" into user-required shapes and sizes; it can recover to the original shape and size automatically once the temperature exceeds the thermal transition temperature (Figure 7). This FSSC can be further woven in electronic textiles to fabricate smart clothes for flexible electronic devices. 


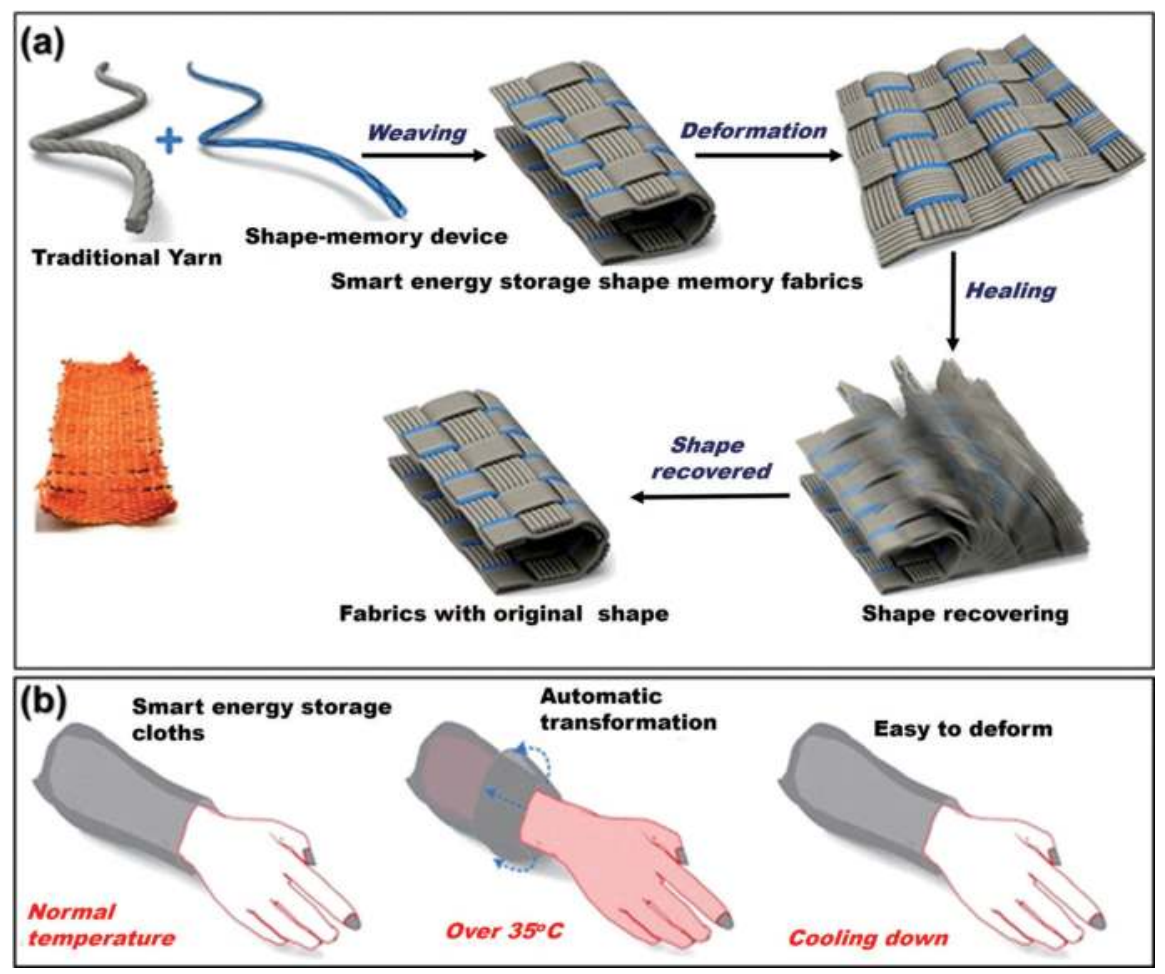

Figure 6. (a) Schematic demonstration of the shape memory SC woven with traditional fabric and the fabrication of a shape-recoverable smart textile and (b) schematic demonstration of this smart shape-memory textile used in a smart cloth (reproduced with permission from Ref. [38]).

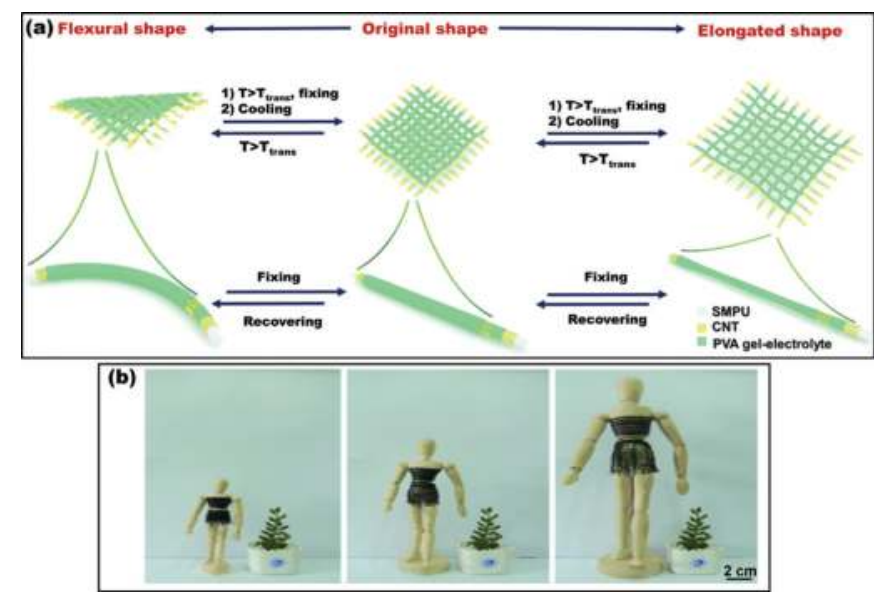

Figure 7. (a) Schematic of the FSSC and the fabricating textile that are reversibly altered into flexural or elongated states and recovered to the original shape and (b) photographs of the smart clothes fabricated by FSSC which were "frozen" into different shapes and sizes (reproduced with permission from Ref. [39]). 


\section{Conclusion}

SMPs provide potential and enormous current opportunities in the textile industry. Textiles with SMPs can move or change their shapes, achieving different 3D forms in garments, enhancing their aesthetic appeal. Window curtains or screens with SMPs can open and close intelligently under environment stimulation. The microstructure or macrostructure changes in smart clothing in response to stimuli are a good means for achieving heat and moisture management of human bodies with a feeling of comfort. The change of fabric configuration can also be used for protection against extreme environments. With the rapid development of SMPs and novel strategies for integrating SMPs into textiles, it is anticipated that the research into smart textiles with SMPs will grow in multiple dimensions as a result of their promising potential applications. In the future, textiles may perform functions that are much more significant, far beyond what is being achieved at present.

\section{Author details}

Suman Thakur

Address all correspondence to: sumanchem89@gmail.com

Institute of Textiles and Clothing, The Hong Kong Polytechnic University, Hung Hom, Hong Kong

\section{References}

[1] Thakur S, Karak N. A tough, smart elastomeric bio-based hyperbranched polyurethane nanocomposites. New Journal of Chemistry. 2015;39(3):2146-2154. DOI: 10.1039/ C4NJ01989J

[2] Yang B, Huang WM, Li C, Li L. Effects of moisture on the thermomechanical properties of a polyurethane shape memory polymer. Polymer. 2006;47:1348-1356. DOI: 10.1016/j. polymer.2005.12.051

[3] Yang G, Wan X, Liu Y, Li R, Su Y, Zeng X, Tang J. Luminescent poly(vinyl alcohol)/ carbon quantum dots composites with tunable water-induced shape memory behavior in different $\mathrm{pH}$ and temperature environments. ACS Applied Materials and Interfaces. 2016;8(50):34744-34754. DOI: 10.1021/acsami.6b11476

[4] Jiang HY, Kelch S, Lendlein A. Polymers move in response to light. Advanced Materials. 2006;18:1471-1475. DOI: 10.1002/adma.200502266

[5] Cho G. Smart Clothing: Technology and Applications. 1st ed. Boca Raton: CRC Press; 2009

[6] Cho G, Lee S, Cho J. Review and reappraisal of smart clothing. The International Journal of Human-Computer Interaction. 2009;25:582-617. DOI: 10.1080/10447310902997744 
[7] Gu JF, Gorgutsa S, Skorobogatiy M. Soft capacitor fibers using conductive polymers for electronic textiles. Smart Materials and Structures. 2010;19:115006. DOI: 10.1088/0964-1726/19/11/115006

[8] Tao X. Wearable Electronics and Photonics. 1st ed. Boca Raton: CRC Press; 2005

[9] Zheng NG, Wu ZH, Lin M. Infrastructure and reliability analysis of electric networks for E-textiles. IEEE Transactions on Systems, Man, and Cybernetics, Part C. 2010;40(1):36-51. DOI: 10.1109/TSMCC.2009.2031497

[10] Gu JF, Gorgutsa S, Skorobogatiy M. Soft capacitor fibers for electronic textiles. Applied Physics Letters. 2010;97:133305. DOI: 10.1063/1.3488351

[11] Kongolo D. Luminescent textiles. WIPO Patent Application WO/2008/148138; 2008

[12] Sayed I, Berzowska J, Skorobogatiy M. Jacquard-woven photonic bandgap fiber displays. Research Journal of Textile and Apparel. 2010;14:97-105

[13] Studstill K. Emotion Sensing Dress Releases Mood Driven Scents [Internet]. 2010. Available from: www.psfk.com/2010/01/emotion-sensing-dress-releases-mood-drivenscents.html

[14] Qi K, Chen X, Liu Y. Facile preparation of anatase/SiO2 spherical nanocomposites and their application in self-cleaning textiles. Journal of Materials Chemistry. 2007;17:35043508. DOI: 10.1039/B702887C

[15] Hu J, Meng H, Li G, Ibekwe SI. A review of stimuli-responsive polymers for smart textile applications. Smart Materials and Structures. 2012;21(5):053001. DOI: 10.1088/0964-1726/21/5/053001

[16] Wang, S. X., Li, Y., Tokura. Effect of Moisture management on functional performance of cold protective Clothing. Text. Res. J.. 2007;77:968-980. DOI: 10.1177/0040517507083552

[17] Crespy D, Rossi R. Temperature-responsive polymers with LCST in the physiological range and their applications in textiles. Polymer International. 2007;56:1461-1468. DOI: 10.1002/pi.2277

[18] Shanmuganathan K, Capadona JR, Rowan SJ, Weder C. Biomimetic mechanically adaptive nanocomposites. Progress in Polymer Science. 2010;35:212-222. DOI: 10.1016/j. progpolymsci.2009.10.005

[19] Mondal S, Hu JL. A novel approach to excellent UV protecting cotton fabric with functionalized MWNT containing water vapor permeable PU coating. The Journal of Applied Polymer Science. 2007;103(5):3370-3376. DOI: 10.1002/app.25437

[20] Hu J. Smart polymers for textile applications. In: Aguilar MR, Román JS, editors. Smart Polymers and Their Applications. 1st ed. Cambridge, UK: Woodhead Publishing; 2014. pp. 437-475

[21] $\mathrm{Hu}$ J, Lu J. Memory polymer coatings for smart textiles. In: Hu J, editor. Active Coatings for Smart Textiles. 1st ed. Cambridge, UK: Woodhead Publishing; 2016. pp. 11-34 
[22] Liu Y, Chung A, Hu JL, Lu J. Shape memory behavior of SMPU knitted fabric. Journal of Zhejiang University SCIENCE A. 2007;8:830. DOI: 10.1631/jzus.2007.A0830

[23] Li Y, Chung SP, Chan L, Hu JL. Characterization of shape memory fabrics. Textile Asia. 2004;35:32-37

[24] Zhong Q, Chen YY, Guan SL, Fang QS, Chen T, Müller-Buschbaum P, Wang JP. Smart cleaning cotton fabrics cross-linked with thermo-responsive and flexible poly(2-(2-methoxyethoxy)ethoxyethyl methacrylate-co-ethylene glycol methacrylate). RSC Advances. 2015;5:38382-38390. DOI: 10.1039/C5RA03256C

[25] Yang H, Zhu H, Hendrix MMRM, Lousberg NJHGM, de With G, Esteves ACC, Xin JH. Temperature-triggered collection and release of water from fogs by a sponge-like cotton fabric. Advanced Materials. 2013;25:1150-1154. DOI: 10.1002/adma.201204278

[26] Chen S, Hu J, Yuen C, Chan L. Novel moisture-sensitive shape memory polyurethanes containing pyridine moieties. Polymer. 2009;50:4424-4428. DOI: 10.1016/j. polymer.2009.07.031

[27] Luo HS, Hu J, Zhu Y. Polymeric shape memory nano-composites with heterogeneoustwin-switches. Macromolecular Chemistry and Physics. 2011;212:1981-1986. DOI: 10.1002/macp.201100292

[28] Lutz JF, Hoth A. Preparation of Ideal PEG analogues with a tunable thermosensitivity by controlled radical copolymerization of 2-(2-methoxyethoxy)ethyl methacrylate and oligo(ethylene glycol) methacrylate. Macromolecules. 2006;39(2):893-896. DOI: 10.1021/ ma0517042

[29] ter Schiphorst J, van den Broek JM, de Koning T, Murphy JN, Schenning APHJ, Esteves ACC. Dual light and temperature responsive cotton fabric functionalized with a surface-grafted spiropyran-NIPAAm-hydrogel. The Journal of Materials Chemistry A. 2016;4:8676-8681. DOI: 10.1039/C6TA00161K

[30] AlliedSignal. Introduces New Fiber to Boost Seatbelt Safety [Internet]. 1999. http://www. accidentreconstruction.com/news/jul99/070699a.html

[31] Hu J, Liu W, Liu B. Fabric-supported chitosan modified temperature responsive PNIPAAm/PU hydrogel and the use thereof in preparation of facial mask. US patent. 2016; US20060286152

[32] Hu J. Research and Scholarly Activities -Smart Fabrics for Magic Cosmetics [Internet]. 2010. Available from: www.polyu.edu.hk/cpa/profile/06feb/pdf/18-23.reseach.pdf [Accessed: March 12, 2011]

[33] Ribeiro MP, Espiga A, Silva D, Baptista P, Henriques J, Ferreira C, Silva JC, Borges JP, Pires E, Chaves P, Correia IJ. Development of a new chitosan hydrogel for wound dressing. Wound Repair and Regeneration. 2009;17:817-824. DOI: 10.1111/j.1524-475X.2009.00538.x

[34] Petrulyte S. Advanced textile materials and biopolymers in wound management. Danish Medical Bulletin. 2008;55:72-77 
[35] Peng H T, Martineau L, Hung A. Hydrogel-elastomer composite biomaterials: 4. Experimental optimization of hydrogel-elastomer composite fibers for use as a wound dressing. The Journal of Materials Science: Materials in Medicine. 2008;19:1803-1813. DOI: $10.1007 / \mathrm{s} 10856-007-3324-y$

[36] Pathak H. Smart' textile materials: A paradigm shift. Journal of Textile Science and Eng ineering. 2012;2:107. DOI: 10.4172/2165-8064.1000e107

[37] Szejtli J. Past, present, and future of cyclodextrin research. Pure and Applied Chemistry. 2004;76:1825-46

[38] Huang Y, Zhu M, Pei Z, Xue Q, Huang Y, Zhi C. A shape memory supercapacitor and its application in smart energy storage textiles. The Journal of Materials Chemistry A. 2016;4:1290-1297. DOI: 10.1039/C5TA09473A

[39] Deng J, Zhang Y, Zhao Y, Chen P, Cheng X, Peng H. A shape-memory supercapacitor fiber. Angewandte Chemie International Edition. 2015;54:15419-15423. DOI: 10.1002/ anie.201508293 
